\title{
POLÍTICAS PÚBLICAS DE PROMOCIÓN DE ESTILOS DE VIDA SALUDABLE COMO FORMA DE GOBIERNO POR MEDIO DE LA COMUNIDAD
}

\author{
POLÍTICAS PÚBLICAS PARA PROMOVER ESTILOS DE VIDA SAUDÁVEIS
}

COMO FORMA DE GOVERNO ATRAVÉS DA COMUNIDADE C己
PUBLIC POLICIES TO PROMOTE HEALTHY LIFESTYLES AS A FORM
OF GOVERNMENT THROUGH COMMUNITY C己

doi' https://doi.org/10.22456/1982-8918.101406

Cristian Ignacio Cabello Jiménez* <cristian.cabello123@gmail.com>

Fernando A. Valenzuela** <fernando.valenzuela@unab.cl>

\footnotetext{
*Investigador autónomo. Santiago, Chile.

**Universidad Andres Bello. Viña del Mar, Chile.
} Sociología.

\begin{abstract}
Resumen: Basado en un estudio de caso, este artículo avanza en la comprensión de los mecanismos concretos que encauzan procesos de subjetivación en el contexto de políticas públicas de promoción de estilos de vida saludable. Analiza el funcionamiento del Programa Vida Sana, implementado por el Gobierno de Chile en el marco del Sistema Elige Vivir Sano, por medio de entrevistas a beneficiarias y a la profesora responsable de las sesiones de actividad física del programa en una localidad de Santiago. Su análisis sugiere la coexistencia de una racionalidad de la elección, que tradicionalmente ha sido identificada con el discurso de la vida activa, con una racionalidad que enfatiza el cuidado mutuo entre los miembros de una comunidad. Se propone que esto tiene implicancias teóricas para la comprensión de las políticas públicas deportivas y de salud como forma de gobierno por medio de la comunidad.
\end{abstract}

Palabras clave: Políticas Públicas de Salud. Estilo de Vida Saludable. Afecto.
Recibido en: 28-03-2020 Aprobado en: 11-11-2020 Publicado en: 28-12-2020

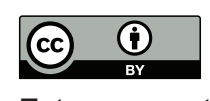

Este es un artículo publicado en Open Access bajo la licencia Creative Commons Atribución 4.0 (CC BY 4.0).

elSSN: $1982-8918$ 


\section{INTRODUCCIÓN}

El modelo de la gubernamentalidad biopolítica neoliberal que fuera esbozado por Michel Foucault $(2006,2007)$ ha probado ser útil para comprender los programas sociales de fomento de estilos de vida saludable que han logrado difusión internacional las últimas décadas como estrategia de prevención y control de enfermedades crónicas no transmisibles (LABOS, 2015). En el trabajo de Foucault, la noción de biopolítica refiere a ensamblajes de discursos, estrategias de intervención y modos de subjetivación relativos a la vitalidad humana colectiva (RABINOW; ROSE, 2006). Bajo el alero de la teoría neoliberal del capital humano (BEDOYA HERNÁNDEZ; CASTRILLÓN ALDANA, 2017; FOUCAULT, 2007; URABAYEN; CASERO, 2018), el gobierno de una población así constituida asume la existencia de sujetos que han de invertir en prácticas de cuidado de sí mismos para maximizar sus retornos en términos de salud y bienestar, enfrentando privadamente los riesgos implícitos en la vida cotidiana (FRAGA, 2005, 2006; GRECO, 1993; LUPTON, 1993, 2003; ROBERTSON, 2001). Como expresa el nombre de la principal iniciativa de fomento de estilos de vida saludable implementada por el Gobierno de Chile, cada uno Elige Vivir Sano (SOTO LAGOS, 2016).

Varios autores inscritos en esta línea de indagación se han enfocado en la experiencia latinoamericana (BAGRICHEVSKY et al., 2010; BAGRICHEVSKY; ESTEVÃO, 2012; CASTIEL; VASCONCELLOS-SILVA, 2006; FERREIRA; CASTIEL; CARDOSO, 2012; FERRER-LUES, 2012, 2013, 2016; FRAGA, 2005, 2006, 2016; PALMA, 2009; PEDRAZ, 2007; PIMENTEL, 2012; PINCHEIRA, 2014; SOTO LAGOS, 2016). Sus análisis se han orientado a comprender las racionalidades que articulan el diseño de esta familia de programas y el aparato prescriptivo en que asientan procesos de subjetivación.

Sin embargo, no se conoce con igual profundidad el despliegue concreto de los programas de fomento de estilos de vida saludable como tecnologías humanas (MILLER, ROSE, 2008) ni los comportamientos morales de los sujetos (ENERGICI, 2016) cuyos cuerpos están en juego: la relación de introyección, negociación o conflicto que los usuarios de estos programas pueden establecer con ella (FERRERLUES, 2016). El objetivo de este artículo es profundizar en la exploración de estas dimensiones por medio del análisis de entrevistas realizadas a 6 usuarias y a la preparadora física del Programa Vida Sana (PVS), perteneciente al sistema Elige Vivir Sano (EVS) implementado por el Gobierno de Chile, en una comuna de la ciudad de Santiago.

Nuestro análisis da cuenta de la coexistencia de una racionalidad de la elección, que tradicionalmente ha sido identificada con el discurso de la vida activa (FRAGA, 2005, 2006; GRECO, 1993; LUPTON, 1993, 2003; ROBERTSON, 2001), con una racionalidad que enfatiza el cuidado mutuo entre los miembros de una comunidad (MOL, 2008). Confirmando estudios previos que han recalcado la necesidad de considerar la dimensión de los afectos en el análisis de políticas sociales, distinguimos tres maneras en que el PVS se conforma como "tecnología afectiva" (MILLION, 2013). Primero, la política social se estructura en base a la generación de vínculos 
afectivos entre agentes del Estado y beneficiarios (ROJAS LASCH, 2018). Segundo, beneficiarias ingresan y permanecen en el programa atendiendo a la condición de salud de familiares y personas cercanas (FERRER-LUES, 2016). Tercero, el mismo programa crea una comunidad en que el cuidado de sí se realiza como cuidado con otros (MENDES; GLEYSE, 2015). Proponemos que esto tiene implicancias teóricas para la comprensión de las políticas sociales deportivas y de salud como forma de "gobierno por medio de la comunidad" (ROSE, 2007).

\section{LA PERSPECTIVA DE LOS ESTUDIOS DE GUBERNAMENTALIDAD}

Este artículo se enmarca en el área de los estudios de gubernamentalidad que se ha desarrollado a partir del trabajo de Foucault (FOUCAULT, 2006, 2007; MILLER; ROSE, 2008; RAMOS ZINCKE, 2012). En este contexto, se entiende por gubernamentalidad,

[. . . ] el conjunto constituido por las instituciones, los procedimientos, análisis y reflexiones, los cálculos y las tácticas que permiten ejercer esa forma bien específica, aunque muy compleja, de poder que tiene por blanco principal la población, por forma mayor de saber la economía política y por instrumento técnico esencial los dispositivos de seguridad (FOUCAULT, 2006, p. 136).

Considerando este contexto teórico, en esta sección expondremos dos distinciones analíticas que permiten fundamentar nuestra aproximación empírica al problema de investigación.

Primero, siguiendo a Miller y Rose (2008), en el análisis de formas de gubernamentalidad es conveniente distinguir entre los programas que producen y problematizan la realidad que será objeto de la razón gubernamental y las tecnologías humanas que encauzan el gobierno de las conductas. En este sentido, los programas de fomento de estilos de vida saludable presuponen la producción de indicadores poblacionales de actividad física y salud que dan forma a la realidad, de manera que puede ser objeto de cálculo. Como han observado estudios previos, en esta dimensión ha sido clave la producción de indicadores de sedentarismo y su enmarcamiento en términos de una epidemia (CAMPOS et al., 2006; FERREIRA; CASTIEL; CARDOSO, 2012; FRAGA, 2016; PALMA, 2009; PIGGIN; BAIRNER, 2016). Por otra parte, se entiende que los programas de fomento de estilos de vida saludable configuran tecnologías humanas de gubernamentalidad: "ensamblajes de personas, técnicas, instituciones, instrumentos para conducir la conducta” (MILLER; ROSE, 2008, p. 16). Específicamente, estos programas configuran estrategias biopolíticas que se orientan a la intervención y maximización de los cuerpos y a su integración en sistemas eficientes (RABINOW; ROSE, 2006). Aquí nos interesará profundizar en la descripción de esta dimensión tecnológica, de ensamblaje de actores heterogéneos con orientación al gobierno de las conductas.

Una segunda distinción analítica es de relevancia para nuestro estudio. Siguiendo a Energici (2016), en los estudios de gubernamentalidad se puede distinguir entre los mecanismos que dan forma a un tipo de población y los procesos de subjetivación, entendidos éstos como programas y tecnologías que dan forma a una subjetividad particular. En el caso que nos interesa aquí, las estadísticas de 
salud y la patologización de la obesidad y el sedentarismo contribuyen a dar forma a una población en riesgo cuyas conductas son conducidas por medio de programas de fomento de estilos de vida saludable. En este artículo buscamos profundizar en la dimensión de los procesos de subjetivación, donde es posible distinguir entre los códigos morales implicados en esta tecnología específica y los comportamientos morales desplegados por los individuos que participan en ella. Es decir, entre "el aparato prescriptivo donde se construyen ciertas formas de ser" (ENERGICl, 2016, p. 36) y la relación de los individuos con los programas y las tecnologías que definen formas de ser.

Diversos estudios críticos han evidenciado que los programas de fomento de estilos de vida saludable implementados en las últimas décadas se orientan a la producción de una subjetividad que corresponde a la figura del empresario de sí mismo (FOUCAULT, 2007): individuos que deciden invertir en sí mismos a través de la modificación de sus hábitos y estilos de vida para asegurar su bienestar ante los riesgos implicados en su vida cotidiana (FERRER-LUES, 2012; FRAGA, 2005, 2006; LUPTON, 2003; PEDRAZ, 2007; PINCHEIRA, 2014; SOTO LAGOS, 2016). En este artículo nos interesará profundizar en el análisis de los comportamientos morales de los sujetos cuyos cuerpos están en juego: la relación de introyección, negociación o conflicto que pueden establecer con los aparatos prescriptivos implicados en esta estrategia biopolítica.

\section{EL PROGRAMA VIDA SANA}

El Gobierno de Chile creó el programa Elige Vivir Sano (EVS) en reacción a una realidad que él mismo había contribuido a producir a través de encuestas. Desde sus mediciones en 2003 y 2006, tanto la Encuesta Nacional de Salud (ENS) como la Encuesta Nacional de Hábitos de Actividad Física y Deportes (ENHAFD) sugieren que más de cuatro quintos de la población adulta de Chile es sedentaria (CHILE, 2017, 2018). Al darse a conocer los resultados de la Encuesta Nacional de Salud de 2010, el gobierno de Chile desarrolló el programa EVS, que llama a cada persona a invertir en su salud (ARUMBURU, 2015). En 2013, esta iniciativa se amplió y logró estabilidad en el presupuesto de la nación bajo la forma del Sistema Elige Vivir Sano (Ley 20.679):

[. . . ] un modelo de gestión constituido por políticas, planes y programas elaborados y ejecutados por distintos organismos del Estado, destinados a contribuir a generar hábitos y estilos de vida saludables y a prevenir y disminuir los factores y conductas de riesgo asociados a las enfermedades no transmisibles. (CHILE, 2013, p. 2)

Sus objetivos han sido establecidos en la ley número 20.670 (CHILE, 2013): 1) fomentar la alimentación saludable; 2) potenciar la actividad física; 3) difundir los beneficios de realizar actividades en familia; y 4) promover el contacto con la naturaleza, el respeto al medio ambiente y la vida al aire libre.

Diversos estudios han evidenciado la racionalidad que otorga sentido y orienta al EVS como estrategia biopolítica: tanto su fundamentación en estadísticas poblacionales como su orientación a la producción de un sujeto que cuida de 
sí ante los riesgos implicados en su vida cotidiana (FERRER-LUES, 2012, 2013; PINCHEIRA, 2014; SOTO LAGOS, 2016). Aquí nos interesará más bien profundizar en el análisis de su dimensión tecnológica, de ensamblaje de actores heterogéneos con orientación al gobierno de las conductas, y de los comportamientos morales de sus usuarios.

Para ello, nos enfocaremos en uno de sus programas, llamado Vida Sana (PVS), cuyo propósito es, "Contribuir a reducir la incidencia de factores de riesgo de desarrollar diabetes mellitus e hipertensión arterial a través de una intervención en los hábitos de alimentación y en la condición física dirigida a niños, niñas, adolescentes, adultos y mujeres post-parto" (CHILE, 2015, p. 7).

El PVS contempla una intervención de 12 meses que incluye atenciones individuales con médicos, nutricionistas y psicólogos, talleres grupales realizados por duplas de nutricionistas y psicólogos, sesiones guiadas de actividad y ejercicio físicos, y exámenes de glicemia y perfil lipídico (CHILE, 2015). Para ello, considera el uso de recursos de cada CESFAM y otros que puedan estar disponibles en su localidad.

Los criterios de ingreso y los objetivos específicos del PVS difieren entre los distintos grupos a los cuales está orientado (CHILE, 2015). Para ingresar al programa, los usuarios deben cumplir alguno de los siguientes criterios de ingreso: diagnóstico nutricional integrado de sobrepeso u obesidad, perímetro de cintura aumentado (población entre 6 y 64 años), o diagnóstico de pre-diabetes o pre-hipertensión (población entre 19 y 64 años). Por su parte, los objetivos específicos contemplan mejorar o mantener la condición nutricional, mejorar la condición física, en términos de capacidad motora (niños y niñas de 2 a 5 años), funcional y muscular (población entre 6 y 64 años), mejorar la condición de pre-diabetes y pre-hipertensión (población entre 6 y 64 años), y asistir a las sesiones de actividad física.

Las sesiones guiadas de actividad y ejercicio físicos son un componente central del PVS (CHILE, 2015). Se contemplan 2 a 3 sesiones semanales de actividad física, sumando un total de 12 horas mensuales por 12 meses. A diferencia de los demás profesionales que participan en el programa, cuyas intervenciones están programadas en intervalos no menores a tres meses, los profesionales a cargo de las sesiones de actividad física tienen un contacto cotidiano con los beneficiarios.

\section{MÉTODOS}

Exploraremos las experiencias de actores vinculados a la ejecución del Programa Vida Sana (PVS) del Ministerio de Salud (MINSAL) del Gobierno de Chile en uno de los Centros de Salud Familiar (CESFAM) administrados por la Corporación Municipal de La Florida en la ciudad de Santiago. Este centro de salud ha ejecutado el PVS desde 2014. Al momento de realizar nuestras observaciones en 2018, el programa tenía un total aproximado de 150 usuarios en esta localidad, en su mayoría mujeres. ${ }^{1}$

1 El perfil histórico general de usuarios del programa ha sido trazado en el informe preparado por Daigre, FerrerLues, y Heynig Pepper (2016). 
Privilegiando estrategias de análisis del discurso basadas en fuentes documentales, estudios previos han dado cuenta de la configuración de programas de fomento de estilos de vida saludable, y del PVS en particular, como estrategia biopolítica neoliberal (BAGRICHEVSKY et al., 2010; BAGRICHEVSKY; ESTEVÃO, 2012; CASTIEL; VASCONCELLOS-SILVA, 2006; FERREIRA; CASTIEL; CARDOSO, 2012; FERRER-LUES, 2012, 2013, 2016; FRAGA, 2005, 2006, 2016; PALMA, 2009; PEDRAZ, 2007; PIMENTEL, 2012; PINCHEIRA, 2014; SOTO LAGOS, 2016). Esta ha sido una estrategia adecuada para develar y criticar el aparato prescriptivo que, orientado a la producción de una subjetividad particular, opera como regla de formación de los enunciados en los que toma forma esta familia de programas deportivos y de salud. Este artículo sigue una estrategia metodológica complementaria para explorar el PVS como tecnología humana (MILLER; ROSE, 2008; ROSE, 1998) y los comportamientos morales desplegados por los sujetos cuyos cuerpos están en juego (ENERGICl, 2016).

Como principal técnica de producción de datos se utilizó la entrevista semiestructurada (SEIDMAN, 2013), con un total de siete, realizadas entre octubre de 2017 y mayo de 2018: una a la preparadora física y seis a beneficiarias del PVS en un CESFAM de la comuna de La Florida, Región Metropolitana. Las entrevistas se realizaron de manera presencial y tuvieron una duración promedio de 40 minutos cada una. Tres entrevistas se realizaron a la hora de término de las sesiones de preparación física de las beneficiarias, dos durante las sesiones de preparación física a las que asistían sus hijos como parte del mismo programa, y una en el CESFAM. Todas las entrevistas fueron realizadas previa presentación y firma de un formulario de consentimiento informado. Con el objetivo de caracterizar las sesiones de preparación física, las entrevistas fueron complementadas con cuatro experiencias no estructuradas de observación no participante, con una duración promedio de 60 minutos cada una (FLORES GUERRERO, 2009)2.

Como criterio general de inclusión en el estudio, se estableció que los beneficiarios debían ser adultos y haber participado en el programa en el sitio seleccionado durante el ciclo 2017. A partir de ello, su selección se orientó según un criterio reputacional (MILES; HUBERMAN, 1994; TEDDLIE; YU, 2007), siguiendo la recomendación de la preparadora física como informante clave y atendiendo a un criterio de saturación de categorías de análisis. Algunos datos de caracterización de las entrevistadas son relevantes para contextualizar los resultados que se comunican. Coincidiendo con el perfil histórico de usuarios del programa, las entrevistadas fueron mujeres de estrato socio-económico medio y medio bajo (DAIGRE; FERRER-LUES; HEYNIG PEPPER, 2016). Si bien se desconoce la distribución etaria de usuarias en el tramo de 20 a 64 años, las entrevistadas presentaron un perfil relativamente homogéneo, cuya edad supera los 50 años. Sus ocupaciones se vinculan a tareas de cuidado: trabajo doméstico propio o de terceros, y cuidadoras de niños o personas mayores. De las seis entrevistadas, sólo tres declararon haber ingresado al programa debido a una condición de salud propia: diabetes, hipertensión arterial y sobrepeso

\footnotetext{
2 Siguiendo a Flores Guerrero (2009), llamamos experiencias no estructuradas no participantes a observaciones de carácter descriptivo que permiten caracterizar el grupo, lugar, ambiente y dar primeras impresiones sobre un fenómeno estudiado.
} 
(2), y depresión (1). De las tres restantes, dos mencionaron como principal motivación el poder realizar actividad física y tener tiempo para sí mismas. Cuatro entrevistadas mencionaron entre sus motivaciones principales apoyar a familiares que, en su opinión, se beneficiarían de la adquisición de hábitos de vida saludable. Cinco entrevistadas declararon no realizar ejercicio físico con anterioridad a su ingreso al programa. Una declaró realizar actividad física sólo ocasionalmente. A la fecha de realización del estudio, las entrevistadas habían participado en el programa durante varios años (4 en promedio), lo que denota la existencia de lazos duraderos entre ellas.

Se implementó una estrategia de análisis cualitativo del discurso (SAYAGO, 2014) inspirada por la arqueología del saber esbozada por Foucault (2013), donde los discursos son entendidos como reglas de formación de enunciados que posibilitan la aparición de objetos y posiciones de subjetividad. Cada entrevista fue transcrita y sometida a un proceso de codificación abductivo, donde se etiquetaron y desagregaron pasajes que realizan categorías discursivas, tanto deducidas del marco teórico como emergentes (SAYAGO, 2014). Los pasajes fueron luego reagregados siguiendo dos estrategias (SEIDMAN, 2013): una diacrónica que reconstruyó temporalmente las trayectorias de participación de las beneficiarias en el programa, permitiendo situar las vivencias y decisiones referidas por ellas en contextos vitales más amplios; y una asincrónica, orientada a la comparación del valor que asumen los tópicos de categorización en distintas entrevistas. Los extractos incluidos en este artículo fueron escogidos por representar más claramente el sentido de cada categoría.

La entrevista a la preparadora física se orientó a la descripción y comprensión de las prácticas que diversos actores llevan a cabo como parte de la realización del programa en este sitio. Atender al relato de las prácticas, buscando entender éstas a luz de los problemas y tensiones que la preparadora física señala experimentar, permite explorar el programa como ensamblaje de elementos heterogéneos (VALENZUELA; RAMOS ZINCKE, 2015): “[. . .] personas, técnicas, instituciones, instrumentos para conducir la conducta" (MILLER; ROSE, 2008, p. 16). En el análisis de la entrevista a la preparadora física se codificaron extractos relativos a tres dimensiones: caracterización de su rol (códigos emergentes referidos a funciones de articulación y enrolamiento, y a su carácter externo), dificultades experimentadas (códigos emergentes referidos a dificultades de financiamiento, coordinación e implementos), y relación con las usuarias (código emergente referido a afección).

Por su parte, las entrevistas a usuarias buscaron observar la relación de introyección, negociación o conflicto que ellas establecen con los aparatos prescriptivos implicados en esta estrategia biopolítica (ENERGICI, 2016). Para ello, se centraron tanto en su descripción de aspectos procedimentales (como su trayectoria de introducción al programa y problemas de índole práctica), como en la forma que asumen en sus expresiones los elementos prescriptivos cuya aparición ha sido posibilitada por el discurso de la vida saludable como parte de un régimen de gubernamentalidad, según ha sido delineado por los estudios previos que hemos comentado: en particular, un sujeto que asume el cuidado de sí ante los riesgos implícitos en su vida cotidiana. En su análisis se codificaron extractos referidos a tres dimensiones definidas de antemano: discurso de vida sana (códigos emergentes vida 
sana como discurso global y práctica deportiva como nueva normalidad), aspectos procedimentales (códigos emergentes acceso, acompañamiento profesional y técnico), apropiación de hábitos (códigos emergentes actividad física y alimentación). A partir del análisis se codificaron extractos referidos a la afectividad y a las relaciones comunitarias como dimensión emergente (códigos emergentes pertenencia, afecto y responsabilidad comunitaria).

\section{RESULTADOS}

Expondremos los resultados en dos secciones. La primera explora los desafíos identificados por la preparadora física en el proceso de implementación del programa y el sentido que otorga a la relación que ha establecido con las beneficiarias. La segunda sección expone las experiencias de las usuarias, enfatizando el sentido que otorgan a su participación en el programa.

\subsection{DEL LADO DE LA PROFESORA}

La preparadora física ocupa una posición central en el PVS en el sitio observado. Si bien formalmente sólo es responsable de las sesiones de actividad física y ejercicio, en la práctica articula a distintos actores que participan en el programa, tanto internos como externos al CESFAM: asegura el acceso a recursos; recluta beneficiarios; y apoya la coordinación de sus sesiones con nutricionistas, psicólogos y médicos. En su rol, construye una relación de confianza con las usuarias, que le permite atender a sus situaciones individuales y realizar acomodos que posibilitan su continuidad en el programa.

Desde la perspectiva de la profesora, el trabajo de los preparadores físicos sostiene el funcionamiento del PVS. En sus palabras, "todo depende del profesor. $\mathrm{Si}$ el profesor no se mueve, prácticamente el programa no funciona" (Entrevista Preparadora Física). Que el profesor "se mueva" significa que, junto con realizar las sesiones de actividad física y las correspondientes evaluaciones siguiendo el protocolo del MINSAL, debe facilitar procesos y gestionar la disponibilidad de recursos críticos para el programa.

Como se ha mencionado, el programa contempla la utilización de recursos externos al centro de salud que lo ejecuta, que puedan estar disponibles en su localidad. En el caso observado, ha recaído en la profesora la responsabilidad de asegurar la disponibilidad de algunos de estos recursos, como una sala de la junta de vecinos que es ocupada para realizar las sesiones de actividad física: "Yo como profesional soy la que va a la junta de vecinos y consigue la cancha. Pero no hay algo que diga, ya, éste es el programa, acá están todos los implementos para que el profesional pueda trabajar. Eso no pasa." (Entrevista Preparadora Física). Ella interpreta esta situación en términos de una inadecuada coordinación entre el CESFAM y otras dependencias de la corporación municipal, que redunda en una insuficiente disponibilidad de espacios e instrumentos deportivos.

Si bien el programa contempla como única vía de ingreso la derivación por parte de personal médico, nutricionista o instituciones educacionales, su mecanismo 
de enrolamiento más efectivo estaría igualmente en manos de la profesora. Ella improvisa estrategias de difusión en espacios concurridos por su público objetivo, como ferias, colegios, jardines infantiles, y dependencias del servicio de salud: "Donde está toda la gente es en la feria. Entonces yo voy con un papel, y voy a las ferias a publicitar el Programa Vida Sana. También voy a los colegios. Voy a los jardines e ingreso panfletos. Y es la única manera de que llegue la gente" (Entrevista Preparadora Física).

En su experiencia, esto genera un encuentro personal inicial que marca la participación de cada beneficiaria en el programa: "Ninguna de las personas de Vida Sana ha entrado al programa directamente. Todas han entrado porque yo de alguna forma me las topé en la calle, en la feria, en el jardín, en el consultorio, en urgencias, etc. Y a ellos les doy toda la información" (Entrevista Preparadora Física).

Partiendo de este encuentro personal inicial, ella construye un lazo de confianza con las beneficiarias, a quienes llama afectuosamente "las chicas". Se puede esperar que esto sea, al menos parcialmente, una consecuencia de la mayor frecuencia de contacto que los preparadores físicos tienen con las beneficiarias. Sin embargo, no se debe subestimar el trabajo que la profesora realiza en este plano. Entre otras estrategias, ha aprovechado los nuevos medios de comunicación digital para crear espacios de comunicación con las usuarias: en particular, administra un grupo de WhatsApp - una plataforma gratuita de mensajería instantánea basada en la red de telefonía móvil - llamado Vida Sana. Por este medio, comparte ejercicios y responde consultas, generando una relación que es descrita en términos de profesionalismo y amabilidad: "En el grupo les mando ejercicios o ellos me mandan sus dudas. Entonces se genera un lazo bastante profesional y de amabilidad, por lo que no existe ningún problema." (Entrevista Preparadora Física).

Esta relación posiciona a la profesora como punto de contacto clave entre la población de usuarios y los recursos del PVS, permitiéndole buscar soluciones a dificultades que eventualmente podrían significar una interrupción indeseada de la práctica de actividad física y, por lo tanto, un fracaso en el intento de generar un hábito. Esto suele tomar la forma de una mediación entre las beneficiarias y el personal administrativo o de salud, para conseguir una atención oportuna: "[...] las chicas me cuentan todo a mí, sus problemas. Y ahí, si ellas me cuentan que no están viniendo por algún problema dental, o por dolores, o alguna enfermedad, yo me voy consiguiendo las horas para que puedan recuperarse y volver a realizar ejercicio." (Entrevista Preparadora Física). Esta mediación, sin embargo, no es siempre exitosa, lo que puede traer consecuencias negativas en el progreso de las beneficiarias.

En el relato de la profesora esta función de mediación toma una forma contradictoria. Por una parte, en línea con el objetivo principal del PVS, la preocupación central de la profesora es que estén dadas las condiciones para que las beneficiarias puedan construir hábitos de actividad física y alimentación, interiorizando de este modo la responsabilidad por asegurar su bienestar. Por eso, uno de los mayores riesgos para el éxito del programa yacería en la interrupción de las rutinas de ejercitación. Por otra parte, paradójicamente, el mismo centro de salud que ejecuta el 
programa puede causar dicha interrupción. Así, el trabajo de mediación toma la forma de una lucha continua contra esta institución.

La profesora resiente en mayor medida problemas operacionales que atribuye al esquema de financiamiento del PVS. Debido a un supuesto retraso en el traspaso de fondos desde el MINSAL, al momento de realizar nuestra entrevista, en abril de 2018, el inicio del programa aún no había sido confirmado. Quienes habían participado en el programa hasta diciembre de 2017 ya sumaban tres meses sin acompañamiento nutricional ni entrenamiento físico supervisado, afectando el principal objetivo declarado del programa: contribuir a la generación de hábitos de vida saludable.

\title{
5.2 DEL LADO DE LAS BENEFICIARIAS
}

Las usuarias identifican su ingreso al programa Vida Sana con un nuevo contexto cultural y vital, en el que se valora más la práctica deportiva y la alimentación saludable. Antes, el deporte habría sido considerado una práctica exclusiva de personas jóvenes y talentosas. Por lo mismo, reconocen en retrospectiva que para ellas no solía ser importante llevar lo que ahora consideran una vida sana. Hoy, en cambio, en todas partes se hablaría de vida sana, comer sano, realizar ejercicio. Con su ingreso al programa, estas prácticas adquieren saliencia:

\begin{abstract}
Ahora ya están hablando por la tele todo lo que es la salud. Y por eso ahora la gente se motiva también. Se empezó a motivarse. Y programas que uno ve. Entonces yo creo que todas esas cosas se empiezan uno a motivarse, de que es bueno esto. (Entrevista Usuaria 1).
\end{abstract}

Junto a ello, perciben una normalización de la práctica deportiva: "Hay gente que, si le da vergüenza hacer ejercicio, ahora es más normal. Ahora cualquiera hace". (Entrevista Usuaria 1).

El ingreso al programa provee acceso a facilitadores de la actividad física. En primer lugar, acceden a un espacio con equipamiento deportivo cercano a sus residencias. En este, además, cuentan con una profesional que las instruye, alienta y acompaña en su introducción a la actividad física:

Con la compañera uno podría caminar, pero, más allá de eso, no. Uno no podría hacer algo más. En cambio, con la profesora es más controlado. A mí me gusta que la profesora me diga: 'Ya, tú tienes que hacer esto, o hace esos ejercicios', porque siento que, con la profesora, esto es más estricto y exigente, ordenado. (Entrevista Usuaria 2).

Este es sobre todo un espacio colectivo, donde las usuarias, en su mayoría mujeres dueñas de casa, conforman un grupo de pares. Una de las entrevistadas se refiere a ellas como "las chiquillas": "[...] ahí uno comparte y la pasamos bien con las chiquillas. Uno se libera con ellas, porque somos todas mujeres, compartimos y hablamos las mismas cosas". (Entrevista Usuaria 3). La participación en el programa otorga un sentido de pertenencia y un carácter fuertemente afectivo:

[...] no me motiva hacer ejercicio sola. Si se acaba vida sana, yo iría a otro programa que hay. Entonces tienen que seguir los programas. También la profe siempre motiva. Entonces como que uno le agarra cariño y hacer ejercicio por el ambiente que tenemos acá. Todas somos mujeres, de acá cerca somos la mayoría dueñas de casa y hablamos, y eso po. Yo por eso no haría ejercicio si se acaba esto. Tendría que buscar en otro lado, 
pero no es lo mismo, porque uno ya se acostumbra acá. Además, es gratis. (Entrevista Usuaria 3).

Se conforma un espacio de encuentro en que se cultivan relaciones de amistad que rebasan los límites del programa. La preparadora física, "la profe" o "la tía", provee un espacio donde las rutinas y preocupaciones cotidianas se ponen entre paréntesis: "Yo antes no hacía nada. No bailaba, nada. Sólo me preocupaba de mis hijos y de la casa. $Y$ ahora bailamos, hacemos zumba. A veces salimos a otras actividades con la profe, que son fuera del programa, y eso" (Entrevista Usuaria 3). Para esta entrevistada, el baile y la participación de "la profe" en actividades que no son parte del programa, ratifican la existencia de este grupo de pares con el que se deja de "no hacer nada".

Para ellas, participar en el programa es "salir de la casa": "Es necesario tener una guía y, además, hacer solo es más fome [aburrido]; porque yo podría poner la tele, poner YouTube y ver rutinas de ejercicio, pero así no me gusta y es fome. Por eso es mejor en compañía, salir de la casa y eso". (Entrevista Usuaria 3).

Participar en el programa es mucho más que realizar actividad física de manera supervisada. También es más que incorporar hábitos saludables. La definición biomédica de lo que constituye una vida sana tiende a pasar a un segundo plano, y adquiere centralidad la posibilidad de compartir con pares en actividades que ponen entre paréntesis las preocupaciones y los roles cotidianos, vinculados a responsabilidades domésticas.

Pero esto no quiere decir que el objetivo explícito de llevar una vida sana según la definición biomédica, y su traducción en el cultivo de hábitos de actividad física y alimentación saludable, se dejen de lado. Por el contrario, como observaran estudios previos (FERRER-LUES, 2016), se evidencia una introyección del discurso neoliberal de la responsabilidad personal por alcanzar el bienestar. Bajo esta luz, pequeñas decisiones cotidianas cobran nuevo sentido. Por ejemplo, entrevistadas cuentan con orgullo que han cambiado hábitos de transporte o alimentación:

Es mucho mejor caminar. No caminar cuadras y cuadras, como se dice, pero.... Yo por lo menos prefiero caminar más. [...] a veces en el verano, con otra compañera, nos vamos caminando [...]. Y ni cuenta nos damos que en 20 minutos ya estamos en el metro y nos hace super bien. (Entrevista Usuaria 1).

Para las usuarias, estas decisiones cotidianas son evidencias de que han incorporado aprendizajes, y de que asumen responsabilidad por su salud.

Al mismo tiempo, concordando con lo observado por Ferrer-Lues (2016), en las entrevistas a usuarias se observa que esta responsabilización adquiere un sentido comunitario: varias han ingresado al programa, y han adoptado estas prácticas y hábitos de vida, considerando la condición de salud de sus hijos o nietos. Así se observa en el relato de una entrevistada, que ingresó al programa por su nieto: "era gordito porque no hacía ejercicio en el colegio. Un día me encontré con la tía [la preparadora física], y ella me motivó para traerlo acá" (Entrevista Usuaria 1).

Si bien las usuarias están conscientes de que sus posibilidades para llevar una vida sana se ven influidas por factores que están mayormente fuera de su control 
-derivadas principalmente de su situación laboral y de sus roles domésticos-, ponen énfasis en factores que pueden controlar: participar en sesiones de actividad física tres veces por semana, caminar en lugar de usar el transporte público, tomar agua.

Sin embargo, estos hábitos de vida saludable quedan en un segundo plano. En el centro está el afecto por "las chiquillas" y por "la profe", con quienes se comparte afuera de la casa. Y estos lazos afectivos con otros miembros de una comunidad sostienen el proceso de subjetivación según el modelo de gubernamentalidad neoliberal.

\section{ANÁLISIS Y CONCLUSIONES}

En las últimas décadas, una serie de programas de fomento de estilos de vida saludable han sido propuestos como solución a epidemias de sedentarismo y obesidad, y a la prevalencia de enfermedades crónicas no transmisibles. Aplicando el modelo de la gubernamentalidad biopolítica neoliberal esbozado por Michel Foucault (2006, 2007), diversos estudios previos han mostrado cómo estos programas configuran racionalidades y tecnologías de gubernamentalidad orientadas al gobierno de las conductas por medio de la producción de sujetos que introyectan la responsabilidad de cuidar de sí ante los riesgos implicados en sus vidas cotidianas (BAGRICHEVSKY et al., 2010; BAGRICHEVSKY; ESTEVÃO, 2012; CASTIEL; VASCONCELLOSSILVA, 2006; FERREIRA; CASTIEL; CARDOSO, 2012; FERRER-LUES, 2012, 2013, 2016; FRAGA, 2005, 2006, 2016; PALMA, 2009; PEDRAZ, 2007; PIMENTEL, 2012; PINCHEIRA, 2014; SOTO LAGOS, 2016).

En diálogo con estos estudios, este artículo ha buscado profundizar en la comprensión del ensamblaje de actores heterogéneos (personas, técnicas, instituciones, instrumentos) que articula el Programa Vida Sana, perteneciente al sistema Elige Vivir Sano implementado por el Gobierno de Chile, como tecnología humana (MILLER; ROSE, 2008) y los comportamientos morales que despliegan sus usuarios (ENERGICI, 2016). Para ello hemos analizado entrevistas realizadas a la preparadora física y a 6 usuarias del programa en una comuna de Santiago.

La entrevista a la preparadora física del PVS en este sitio entrega una nueva imagen de los mecanismos que posibilitan la producción de una subjetividad neoliberal. En primer lugar, se observa que su ámbito de responsabilidades rebasa los límites que han sido definidos formalmente. Ella ocupa una función clave en el contacto inicial y en el enrolamiento de beneficiarias, y en la generación de espacios de comunicación que permiten atender a sus dificultades, mediando entre ellas y personal administrativo o de salud del CESFAM. A la base de esta función de mediación está la generación de un sentido de pertenencia a una comunidad y, sobre todo, la construcción de lazos afectivos entre agentes del Estado y beneficiarias. En este sentido, en este sitio es posible reconocer lo que Rojas Lasch ha llamado "lo social asistencializado": un modo de acción estatal donde "afectarse resulta ser también estratégico e incluso profesionalizante" (ROJAS LASCH, 2018, p. 133). Construyendo en base a lo que ha observado esta autora en su análisis de otros tipos de programas, esta manera de estructurar las relaciones entre agentes del Estado 
y beneficiarios de la política social hace posible, efectivamente, la producción de sujetos que introyectan la responsabilidad de alcanzar una vida sana por medio de la creación de hábitos de alimentación y ejercicio físico.

Por su parte, si bien en las entrevistas a usuarias se observa una introyección de este discurso neoliberal de la vida saludable, se confirma al mismo tiempo que ellas expanden la responsabilidad de cuidar de sí para atender los estilos de vida de familiares y personas cercanas (FERRER-LUES, 2016). Más importantemente, se observa que el programa crea una comunidad donde el cuidado de sí se realiza como cuidado con otros (MENDES; GLEYSE, 2015), facilitando la adherencia a las rutinas de actividad física.

De esta manera, nuestro análisis da cuenta de la coexistencia de una racionalidad de la elección, que tradicionalmente ha sido identificada con el discurso de la vida activa (FRAGA, 2005, 2006; GRECO, 1993; LUPTON, 1993, 2003; ROBERTSON, 2001), con una racionalidad que enfatiza el cuidado mutuo entre los miembros de una comunidad (MOL, 2008). La noción de "gobierno por medio de la comunidad", propuesta por Rose (2007), permite conceptualizar este estado de cosas. Como ha mostrado este autor, ya desde el siglo XX, mentalidades y estrategias de gubernamentalidad han pasado a concebir a los sujetos de gobierno como personas que, siendo activas en su propio gobierno, establecen "una relación de lealtad y responsabilidad para con los seres cercanos, con quienes el propio destino está asociado" (2007, p. 117). Proponemos que los comportamientos morales de los usuarios de los programas de fomento de estilos de vida saludable, y del PVS en particular, no pueden ser comprendidos plenamente si no se toman en cuenta los mecanismos propios de esta forma de gobierno. Entre ellas se destaca una dimensión afectiva, en la medida que estos programas asumen que las personas están supeditadas a lazos emocionales de afinidad con miembros de su comunidad local (ROSE, 2007, p. 121). Usando la noción propuesta por Million (2013), proponemos que ellas pueden describirse como "tecnologías afectivas", pues forman parte de un universo de prácticas estatales orientadas a conducir la conducta de poblaciones humanas mediante la gestión de los afectos.

Como tecnología afectiva que realiza una forma de gobierno por medio de la comunidad, el Programa Vida Sana posibilita procesos de subjetivación característicos de contextos de liberalismo avanzado en donde el cuidado de sí se realiza como cuidado con otros, introyectando la responsabilidad de hacer frente a los riesgos implicados en nuestras vidas cotidianas. 


\section{REFERENCIAS}

ARUMBURU, Javier. Cecilia Morel, embajadora de un Chile saludable. Revista Valora, 22 dez. 2015. Disponible en: https://www.revistavalora.cl/cecilia-morel-embajadora-de-un-chilesaludablel. Acceso en: 10 dic. 2020.

BAGRICHEVSKY, Marcos; CASTIEL, Luis David; VASCONCELLOS-SILVA, Paulo Roberto; ESTEVÃO, Adriana. Discursos sobre comportamento de risco à saúde e a moralização da vida cotidiana. Ciência \& Saúde Coletiva, v. 15, n. supl. 1, p. 1699-1708, jun. 2010.

BAGRICHEVSKY, Marcos; ESTEVÃO, Adriana. O imperativo da epidemiologia dos fatores de risco em face das desigualdades sociais: Agenciamentos discursivos sobre o sedentarismo. Revista De Ciências Sociais - Política \& Trabalho, p. 89-102, 18 dez. 2012.

BEDOYA HERNÁNDEZ, Mauricio; CASTRILLÓN ALDANA, Alberto. Neoliberalismo como forma de subjetivación dominante. Dorsal - Revista de Estudios Foucaultianos, v. 3, p. 31-56, 12 dic. 2017.

CAMPOS, Paul; SAGUY, Abigail; ERNSBERGER, Paul; OLIVER, Eric; GAESSER, Glenn. The epidemiology of overweight and obesity: public health crisis or moral panic? International Journal of Epidemiology, v. 35, n. 1, p. 55-60, 1 feb. 2006.

CASTIEL, Luis David; VASCONCELLOS-SILVA, Paulo Roberto. A noção "estilo de vida" em promoção de saúde: um exercício crítico de sensibilidade epistemológica. In: BAGRICHEVSKY, M.; PALMA, A.; ESTEVÃO, A.; DA ROS, M. (eds.). A saúde em debate na educação física. Blumenau: Nova Letra, 2006. v. 2. p. 67-90.

CHILE. Ley $\mathbf{n}^{\circ} \mathbf{2 0 . 6 7 0}$, de 31 de mayo de 2013. Crea el Sistema Elige Vivir Sano, 31 maio 2013. Disponible en: https://www.leychile. $c / / N ? \mathrm{i}=1051410 \& \mathrm{f}=2019-05-02 \& \mathrm{p}=$. . Acceso en: 10 dic. 2020

CHILE. Orientaciones y Lineamientos Programa Vida Sana. Intervención en Factores de Riesgo de Enfermedades No Transmisibles. Chile: Ministerio de Salud ; Subsecretaría de Redes Asistenciales, 2015.

CHILE. Encuesta Nacional de Salud 2016-2017: Primeros Resultados. Chile: Ministerio de Salud; Subsecretaría de Salud Pública, 2017. Disponible en: https://www.minsal.cl/wpcontent/uploads/2017/11/ENS-2016-17_PRIMEROS-RESULTADOS.pdf. Acceso en: 9 sept. 2020.

CHILE. Encuesta Nacional de Hábitos de Actividad Física y Deportes en la Población de 18 años y más. Chile: Ministerio del Deporte; Instituto Nacional del Deporte, 2018. Disponible en: http://www.mindep.cl/wp-content/uploads/2016/07/INFORME-FINALENCUESTA-DEPORTES-COMPLETO_.pdf. Acceso en: 9 sept. 2020.

DAIGRE, María Luisa; FERRER-LUES, Marcela; HEYNIG PEPPER, Elisa. Informe Final de Evaluación. Programa Vida Sana. Intervenciones en Factores de Riesgo de Enfermedades no Transmisibles en Niños, Adolescentes, Adultos y Mujeres Post-Parto. [Santiago: Ministerio de Salud; Secretaría de Redes Asistenciales, 2016. Disponible en: https://www. dipres.gob.cl/597/articles-149542_informe_final.pdf. Acceso en: 10 dic. 2020

ENERGICl, María Alejandra. Propuesta metodológica para un estudio de gubernamentalidad: Los procesos de subjetivación y los mecanismos de regulación poblacional como ejes de análisis para su abordaje empírico. Psicoperspectivas. Individuo y Sociedad, v. 15, n. 2, p. 29-39, 15 jul. 2016. 
FERREIRA, Marcos Santos; CASTIEL, Luis David; CARDOSO, María. Helena Cabral de Almeida. A patologização do sedentarismo. Saúde e Sociedade, v. 21, n. 4, p. 836-847, dez. 2012.

FERRER-LUES, Marcela. ¿La salud como responsabilidad individual? Análisis del concepto de salud en los Programas de Gobierno de la Concertación en Chile. En: CONGRESO DE LAASOCIACIÓN LATINOAMERICANA DE SOCIOLOGÍA, 24, 2013. Acta Científica, 2013.

FERRER-LUES, Marcela. Percepción del discurso de los estilos de vida saludable y su repercusión en el comportamiento de población adscrita al Programa Vida Sana en la comuna de Pedro Aguirre Cerda. Santiago: FONIS, 2016. Disponible en: http://repositorio. conicyt.cl/handle/10533/209694. Acceso en: 10 dic. 2020.

FERRER-LUES, Marcela. Reflexiones en torno a la concepción de salud como responsabilidad individual: Construyendo bioética en salud pública. In: LEÓN CORREA, F. J. (ed.). Bioética y Sociedad en Latinoamérica. Santiago: Fundación Interamericana Ciencia y Vida, 2012. p. 115-122.

FLORES GUERRERO, Rodrigo. Observando Observadores: Una Introducción a Las Técnicas Cualitativas De Investigación Social. Santiago: Eds. Universidad Católica de Chile, 2009.

FOUCAULT, Michel. La arqueología del saber. Buenos Aires: Siglo XXI, 2013.

FOUCAULT, Michel. Nacimiento de la biopolítica. Curso en el College de France (19781979). México: Fondo de Cultura Económica, 2007.

FOUCAULT, Michel. Seguridad, territorio, población. Curso en el College de France (1977-1978). México: Fondo de Cultura Económica, 2006.

FRAGA, Alex Branco. Exercício da informação : governo dos corpos no mercado da vida ativa. 2005. Tese (Doutorado em Educação) - Faculdade de Educação, Universidade Federal do Rio Grande do Sul, Porto Alegre, 2005.

FRAGA, Alex Branco. Promoção da vida ativa: nova ordem físico-sanitária na educação dos corpos contemporâneos. En: BAGRICHEVSKY, M.; PALMA, A.; ESTEVÃO, A.; DA ROS, M. (Eds.). A saúde em debate na educação física. Blumenau: Nova Letra, 2006. v. 2p. 105-119.

FRAGA, Alex Branco. El sedentarismo es... Saúde e Sociedade, v. 25, n. 3, p. 716-720, set. 2016.

GRECO, Monica. Psychosomatic subjects and the 'duty to be well'. personal agency within. Economy and Society, v. 22, n. 3, p. 357-372, ago. 1993.

LABOS, Cristopher. Lifestyle Interventions: The Best Medicine You're Not Using. Medscape, 22 abr. 2015. Disponible en: http://www.medscape.com/viewarticle/843028. Acceso en: 10 dic. 2020.

LUPTON, Deborah. Risk as Moral Danger: The Social and Political Functions of Risk Discourse in Public Health. International Journal of Health Services, v. 23, n. 3, p. 425435, jul. 1993.

LUPTON, Deborah. "Desenvolvendo-me por inteiro": cidadania, neoliberalismo e saúde contemporânea no currículo de Educação Física. Movimento, v. 9, n. 3, p. 11-31, 2003. 
MENDES, Maria Isabel Brandão de Souza; GLEYSE, Jacques. O cuidado de si em Michel Foucault: reflexões para a educação física. Movimento, v. 21, n. 2, p. 507-520, 2015.

MILES, Mathew; HUBERMAN, Michael. Qualitative Data Analysis. Thousand Oaks: SAGE Publications, 1994.

MILLER, Peter; ROSE, Nikolas. Governing the Present: Administering Economic, Social and Personal Life. Cambridge Malden: Polity, 2008.

MILLION, Dian. Therapeutic nations: healing in an age of indigenous human rights. Tucson: The University of Arizona, 2013.

MOL, Annemarie. The Logic of Care: Health and the problem of patient choice. London; New York: Routledge, 2008.

PALMA, Alexandre. Exercício Físico e Saúde; Sedentarismo e Doença: Epidemia, Causalidade e Moralidade. Motriz, v. 15, n. 1, p. 185-191, 2009.

PEDRAZ, Miguel Vicente. Estilo de vida saludable e ideología: dialéctica de la diferencia. Salud Pública de México, v. 49, n. 6, p. 388, dic. 2007.

PIGGIN, Joe; BAIRNER, Alan. The global physical inactivity pandemic: an analysis of knowledge production. Sport, Education and Society, v. 21, n. 2, p. 131-147, 17 fev. 2016.

PIMENTEL, Giuliano Gomes de Assis. O passivo do lazer ativo. Movimento, v. 18, n. 3, p. 299-316, 3 set. 2012.

PINCHEIRA, Iván. Programa gubernamental "Elige Vivir Sano": Cuerpo, salud y felicidad en Chile durante la presidencia de Sebastián Piñera. In: OYANEDEL, J. C.; MELLA, C. (eds.).

Debates sobre el bienestar y la felicidad. Santiago: Ril, 2014. p. 107-128.

RABINOW, Paul; ROSE, Nikolas. Biopower Today. BioSocieties, v. 1, p. 195-217, 2006.

RAMOS ZINCKE, Claudio. El ensamblaje de ciencia social y sociedad. Santiago: Universidad Alberto Hurtado, 2012.

ROBERTSON, Ann. Biotechnology, political rationality and discourses on health risk. Health, v. 5, n. 3, p. 293-309, 2001.

ROJAS LASCH, Carolina. Afecto y cuidado: pilar de la política social neoliberal. Polis (Santiago), v. 17, n. 49, p. 127-149, maio 2018.

ROSE, Nikolas. Inventing Our Selves: Psychology, Power, and Personhood. Cambridge: Cambridge University, 1998.

ROSE, Nikolas. ¿La muerte de lo social? Re-configuración del territorio de gobierno.

Revista Argentina de Sociología, v. 5, n. 8, p. 111-150, 2007.

SAYAGO, Sebastián. El análisis del discurso como técnica de investigación cualitativa y cuantitativa en las ciencias sociales. Cinta de moebio, n. 49, p. 1-10, 2014.

SEIDMAN, Irving. Interviewing as Qualitative Research: A Guide For Researchers in Education \& The Social Sciences. 4. ed. New York; London: Teachers College, 2013.

SOTO LAGOS, Rodrigo Andrés. Sedentarismo, deporte y la presión biopolítica de vivir saludable: análisis del discurso del sistema Elige Vivir Sano en Chile. Movimento, v. 22, n. 2, p. 391-402, 2016. 
TEDDLIE, Charles; YU, Fen. Mixed Methods Sampling: A Typology With Examples. Journal of Mixed Methods Research, v. 1, n. 1, p. 77-100, Jan. 2007.

URABAYEN, Julia; CASERO, Jorge León. La gubernamentalidad biopolítica: de la sociedad de control estatal al liberalismo. Co-herencia, v. 15, n. 29, p. 67-92, Ago. 2018.

VALENZUELA, Fernando Andrés; RAMOS ZINCKE, Claudio. Cómo los maltratos se transforman en 'violencia intrafamiliar'. El recorrido de la performatividad. Revista de Estudios Sociales, v. 51, p. 213-226, 2015. 
Resumo: Com base em um estudo de caso, este artigo avança na compreensão dos mecanismos específicos que canalizam os processos de subjetivação no contexto das políticas públicas para promover estilos de vida saudáveis. Analisa a operação do Programa Vida Sana, implementado pelo Governo do Chile, no âmbito do Sistema Escolha Uma Vida Saudável, por meio de entrevistas com beneficiários e a professora responsável pelas sessões de atividade física do programa em um distrito de Santiago. Sua análise sugere a convivência de uma racionalidade de escolha, que tradicionalmente tem sido identificada com o discurso da vida ativa, com uma racionalidade que enfatiza o cuidado mútuo entre os membros de uma comunidade. Propõe-se que isso tenha implicações teóricas para a compreensão das políticas públicas de esportes e saúde como forma de governo através da comunidade.

Palavras chave: Políticas Públicas de Saúde. Estilo de Vida Saudável. Afeto. Sociologia.

\begin{abstract}
Based on a case study, this article advances in understanding the concrete mechanisms that channel subjectivation processes in the context of public policies to promote healthy lifestyles. It analyzes the operation of the Vida Sana Program, implemented by the Government of Chile within the framework of the Choose Healthy Living System, through interviews with beneficiaries and the teacher responsible for the program's physical activity sessions in a district of Santiago. Its analysis suggests the coexistence of a rationality of choice, which has traditionally been identified with the discourse of active life, with a rationality that emphasizes mutual care among members of a community. The study proposes that it has theoretical implications for understanding sports and health public policies as a form of governing through community.
\end{abstract}

Keywords: Public Health Policy. Healthy Lifestyle. Affect. Sociology. 


\section{LICENCIA DE USO}

Este es un artículo publicado en Open Access bajo la licencia Creative Commons Atribución 4.0 Internacional (CC BY 4.0), que permite su uso, distribución y reproducción en cualquier medio, siempre que la obra original esté correctamente citada. Más información en: http://creativecommons.org/licenses/by-nc/4.0

\section{CONFLICTO DE INTERESES}

Los autores han declarado que no existe conflicto de intereses en este trabajo.

\section{CONTRIBUCIONES DE LOS AUTORES}

Cristian Ignacio Cabello Jiménez: Diseño de investigación. Revisión bibliográfica. Recopilación fuentes de datos secundarias. Diseño y aplicación de instrumentos de observación. Transcripción de entrevistas. Análisis de entrevistas. Escritura de artículo.

Fernando Andrés Valenzuela: Sistematización de literatura. Supervisión y orientación proceso de diseño de investigación, recopilación de fuentes de datos secundarias, diseño y aplicación de instrumentos de observación. Análisis de entrevistas. Escritura de artículo.

\section{APOYO E FINANCIACIÓN}

O presente trabalho foi realizado com apoio da ANID, Gobierno de Chile; Universidad Andrés Bello, Facultad de Educación y Ciencias Sociales.

Los resultados comunicados en este artículo forman parte de un proyecto financiado por el programa FONDECYT Iniciación en la Investigación 11150918, "Telemedicina, controversia y cambio social en Chile. Cómo la telemedicina se construye socialmente y configura la sociedad".

\section{CÓMO HACER REFERENCIA}

CABELLO JIMENEZ, Cristian Ignacio; VALENZUELA, Fernando A.. Políticas públicas de promoción de estilos de vida saludable como forma de gobierno por medio de la comunidad. Movimento (Porto Alegre), v.26, p. e26092, jan./dez. 2020. Disponible en: https://seer.ufrgs.br/Movimento/article/view/101406. Acceso en: [día] [mes abreviado]. [año]. DOI: https://doi.org/10.22456/1982-8918.101406

\section{RESPONSABILIDAD EDITORIAL}

Alex Branco Fraga*, Elisandro Schultz Wittizorecki*, Ivone Job*, Mauro Myskiw*, Raquel da Silveira*

*Universidade Federal do Rio Grande do Sul, Escola de Educação Física, Fisioterapia e Dança, Porto Alegre, RS, Brasil 